\title{
Enhancement of XPS surface sensitivity in nanocrystalline material
}

\author{
Rafal J. Wróbel \\ West Pomeranian University of Technology, Szczecin, Institute of Chemical and Environment Engineering, \\ ul. Pulaskiego 10, 70-322 Szczecin, Poland, e-mail: rwrobel@zut.edu.pl
}

The influence of the particle size on the surface sensitivity in XPS analysis was investigated. Previous reports about such influence were qualitatively only. In this report there are given mathematical description of XPS sensitivity and quantitative results. It was found that influence due to nanometric size on XPS analysis can be noticeable for particles below $15 \mathrm{~nm}$ of diameter and increases dramatically with reduction of the size.

Keywords: XPS, nanomaterials, surface science.

\section{SOLVENT-FREE PRESSURE-SENSITIVE ADHESIVES}

X-ray photoelectron spectroscopy (XPS) is one of the most widely used techniques for surface analysis. During XPS measurement the core electrons are excited by X-ray. The kinetic energy of emitted electrons is unique for given element. Only electrons which have not lost their energy due to the collisions are useful for analysis. Although Xrays can penetrate the bulk of material in micrometric range the photoelectrons without energy loss originate from very few $\mathrm{nm}$. The measure of electron transparency is the inelastic mean free path (IMFP) which is a function of electron energy and material composition. For electrons in typically applied in XPS energy range $(0-1000$ $\mathrm{eV}$ ) the IMFP is $0.5-3 \mathrm{~nm}$. Thus surface sensitivity of this technique is in range $2-5 \mathrm{~nm}^{1}$. This thin layer within the XPS analysis occurs may be inhomogeneous what poses the problem of accurate XPS analysis and is of extreme importance in catalysis. Therefore the great deal of work was devoted for determination of IMFP for different materials especially by Jablonski and Powell ${ }^{2}$. This heterogeneity can be also solved via angle resolved XPS measurements ${ }^{3}$. Electrons collected with angle due to the geometric reasons originate more likely from surface. However angle resolved XPS can be applied for flat samples only like monocrystals etc. Recently it was reported that in case of powders, especially nanopowders, it should be observed the higher sensitivity for surface in comparison to the monocrystals ${ }^{4}$. However in this report there are given neither size dimensions in which the effect should be observed nor the magnitude of increased sensitivity. The goal of this paper is to fill this gap and describe the phenomenon quantitatively.

\section{RESULTS AND DISCUSSION}

In XPS the electrons are attenuated by material through which they have to pass. This attenuation can be described by Lambert-Beer equation ${ }^{5}$ (Eq.1). Let us consider the overlayer A deposited on substrate B. The higher angle $\theta$ the stronger attenuation of substrate B signal can be observed. In normal XPS analysis $\theta$ angle equals $90 \mathrm{deg}$ thus term $\sin (\theta)$ is equal unity.

$I=I_{0} \cdot e^{-\frac{z}{\lambda \cdot \sin \theta}}$

Where: I - intensity of the attenuated signal by overlayer of " $\mathrm{z}$ " thickness, $\mathrm{I}_{0}$ - intensity without attenuation, $\lambda$ - IMFP of electron, $\theta$ - angle between the surface of overlayer and the vector of measured electron.
In Fig. 1 is presented situation for powder particle which has overlayer A on substrate B. In this case one can observe that emission angle in respect to the substrate surface vary for different points of the particle. Therefore in XPS analysis of powders in comparison with flat samples the substrate signal is more attenuated. This is the reason of higher surface sensitivity of powders as it was reported in Ref. 4.

However the Ref. 4 gives qualitatively reasoning only and thus one can not say how strong is phenomenon. To

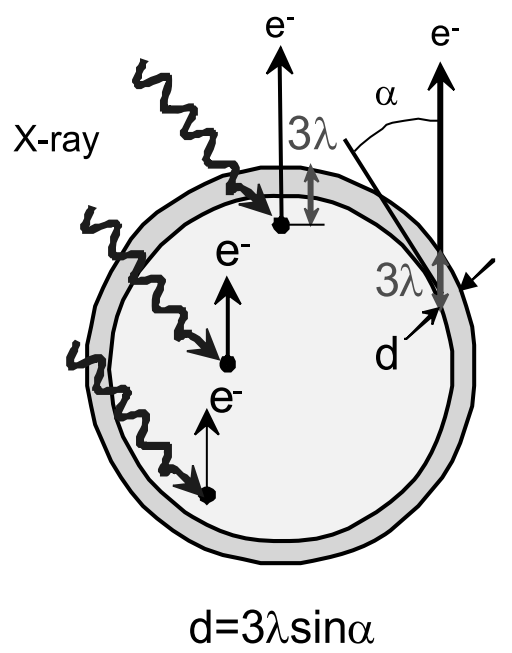

Figure 1. The schematic representation of surface sensitivity enhancement in powder materials based on Ref. 4. Due to the curvature of the surface most of the electrons are emitted with emission angle thus surface sensitivity is increased. The sensitivity increases with decrease of particle size. $3 \lambda-$ represents the depth from most of the signal acquired

overcome this difficulty let us consider situation of spherical particle of substrate $\mathrm{B}$ with overlayer A of thicknes $\mathrm{X}$ (Fig. 2). For the sake of simplicity let us assume that 1 has the same value for both substrate and overlayer.

In this case the total intensity IT of both the substrate $\mathrm{A}$ and the overlayer B can be derived from Eq. 2.

$I_{T}=2 \cdot \pi \cdot I_{0} \int_{-R}^{R} \int_{0}^{\sqrt{R^{2}-h^{2}}} r \cdot e^{-\frac{-h+\sqrt{R^{2}-r^{2}}}{\lambda}} d r \cdot d h$ 


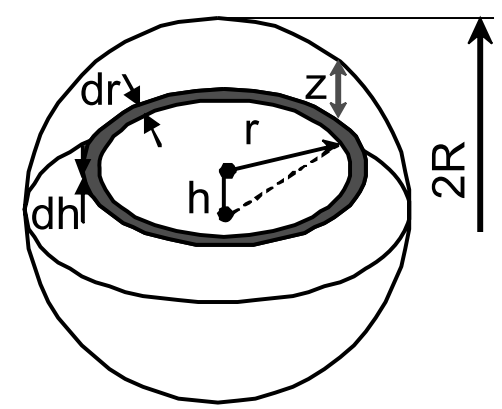

Figure 2. Schema of the spherical particle of substrate A with overlayer B of thickness " $\mathrm{x}$ " (not denoted on the figure). The variables required for integrals are denoted with small letters. $\mathrm{R}$ - radii of the spherical particle, $\mathrm{z}$ - attenuation depth from which the signal originates

Where: $\mathrm{R}$ - radii of the spherical particle; $\mathrm{r}, \mathrm{h}$ - variables (see Fig. 2 for details).

In order to receive the intensity of substrate B one has to decrease borders of integration by thickness of the overlayer $\mathrm{x}$ (Eq. 3).

$I_{B}=2 \cdot \pi \cdot I_{0} \int_{-(R-x)}^{R-x} \int_{0}^{\sqrt{R^{2}-h^{2}}} r \cdot e^{-\frac{-h+\sqrt{R^{2}-r^{2}}}{\lambda}} d r \cdot d h$

Where: $\mathrm{x}$ - thickness of overlayer A.

Based on the Eq. 2 and Eq. 3. one can calculate the intensity of overlayer signal and molar fraction (Eq. 4. Eq. 5. respectively).

$I_{A}=I_{T}-I_{B}$

$X_{A}=\frac{I_{A}}{I_{A}+I_{B}}$

Armed with above given equations one can calculate dependence of overlayer signal of fixed thicknes $\mathrm{x}$ on particle size. The calculations were performed numerically for particle diameters $2,4,8,16,32 \mathrm{~nm}$; thickness x 0.2 and $0.6 \mathrm{~nm}$ and $\lambda-0.5,1.0,2.0 \mathrm{~nm}$. The higher diameters of the particles were also calculated but the results will be not presented due to negligible influence of diameter above $32 \mathrm{~nm}$ on surface sensitivity. The overlayer thickness of $0.2 \mathrm{~nm}$ was choosen as corresponding to thickness of the monolayer of typical atoms on substrate. The thickness $0.6 \mathrm{~nm}$ corresponds to the film of $2-3$ monolayers. Values of 1 for most materials and XPS kinetic energies are typically in range $0.5-3.0 \mathrm{~nm}^{1}$.
In Fig. 3 the dependence of XPS signal overlayer A on partcle size was presented.

One can notice that pronounce enhancement of XPS surface sensitivity occurs for particles of diameter below $10 \mathrm{~nm}$. The enhancement can be as high as $100 \%$ in comparison with coarse particles with the overlayer of the same thickness. However one should be aware that it is also connected with content increase of overlayer phase in the sample. Namely in case of such small nanoparticles the composition of surface starts to be the main factor governing the average composition of the sample. One can also observe the decrease of overlayer signal with increase of $\lambda$. This effect is related with the attenuation of the substrate signal. The higher value of $\lambda$ the smaller is attenuation and thus the smaller signal of overlayer A can be observed. Analogously the thicker overlayer A results in higher attenuation of substrate B.

\section{CONCLUSIONS}

The effect of enhancement of XPS surface sensitivity in nanocrystalline materials was mathematically described. The calculations were performed numerically for particle diameters $2,4,8,16,32 \mathrm{~nm}$; thickness $\mathrm{x} 0.2$ and $0.6 \mathrm{~nm}$ and $\lambda-0.5,1.0,2.0 \mathrm{~nm}$. It was observed that pronounce effect occurs for particles of diameter below $10 \mathrm{~nm}$. The enhancement can be as high as $100 \%$ in comparison with coarse particles with the overlayer of the same thickness.

\section{LITERATURE CITED}

1. Tougaard, S. (2005). XPS for Quantitative Analysis of Surface Nano-structures. Microsc. Microanal. 11(2), 676 - 677. DOI:10.1017/S1431927605500229.

2. Jablonski, A. \& Powell, C.J. (2004). Electron effective attenuation lengths in electron spectroscopies, J. Alloy. Compd. 362, 26 - 32. DOI:10.1016/S0925-8388(03)00558-9.

3. Gunter, P.L.J. (1992). Evaluation of take-off-angle-dependent XPS for determining the thickness of passivation layers on aluminium and silicon. Surf Interface Anal. 19, 161 - 164. DOI: 10.1002/sia.740190131.

4. Suchorski, Y., Wrobel, R., Becker, S., Opalinska, A., Narkiewicz, U., Podsiadly, M. \& Weiss, H. (2008). Surface chemistry of zirconia nanopowders doped with $\operatorname{Pr}_{2} \mathrm{O}_{3}$ : an XPS study, Acta Phys Pol A, 114, 1250150134.

5. Briggs, D. (2005). Surface analysis of polymers by XPS and static SIMS, Cambridge Solid State Science Series, Cambridge University Press.
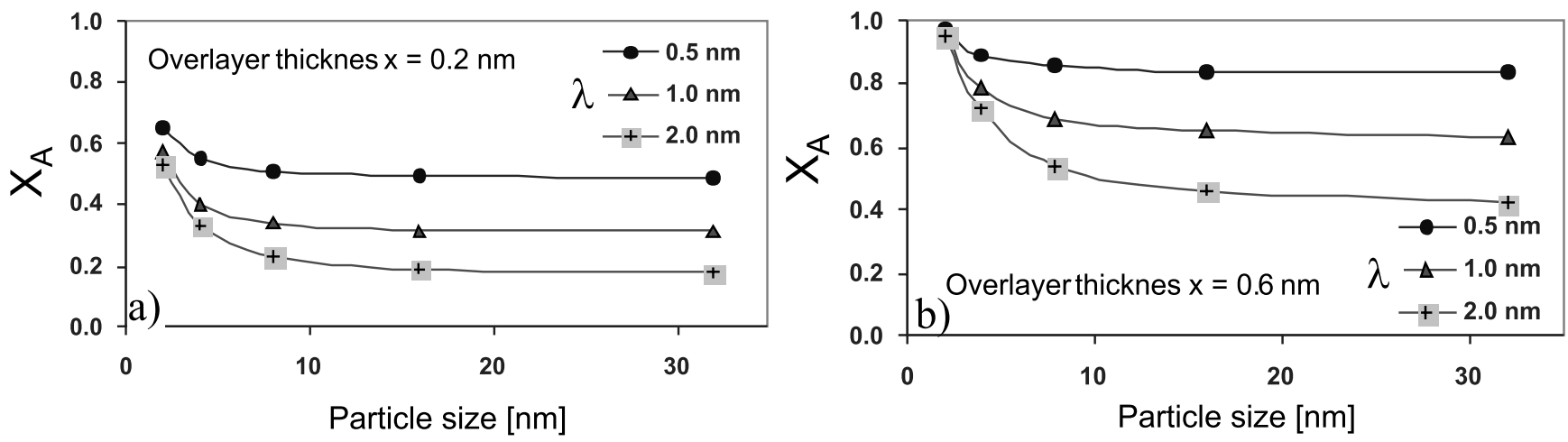

Figure 3. Dependence of XPS signal of overlayer A (as molar fraction $X_{A}$ ) on particle size. Three lines represents three different $\lambda$. a) Thickness of overlayer $0.2 \mathrm{~nm}$ i.e. typical monolayer of atoms. b) Thickness of overlayer $0.6 \mathrm{~nm}$ i.e. $2-3$ monolayers of atoms 